\title{
Vibration Analysis of an Axially Loaded Viscoelastic Nanobeam
}

\author{
Mustafa ARDA ${ }^{a^{*}}$ \\ ${ }^{a}$ Trakya University, Mechanical Engineering Department, Edirne, 22030 TURKEY \\ ${ }^{*}$ E-mail address: mustafaarda@trakya.edu.tr
}

Received date: 09.10 .2018

Accepted date: 02.11 .2018

ORCID numbers of authors:

0000-0002-0314-3950

\begin{abstract}
Vibration of an axially loaded viscoelastic nanobeam has been studied in this paper. Viscoelasticity of the nanobeam has been modeled as a Kelvin-Voigt material. Equation of motion and boundary conditions for an axially compressed nanobeam has been obtained with help of Eringen's Nonlocal Elasticity Theory. Viscoelasticity effect on natural frequency and damping of nanobeam and critical buckling load have been investigated. Nonlocality effect on nanobeam structure in the view of viscoelasticity has been discussed.
\end{abstract}

Keywords: viscoelastic nanobeam, nonlocal elasticity, vibration, axially loaded, buckling.

\section{Introduction}

Nano-sized structures are like carbon nanotubes (CNTs) taken interests of scientists over the years. The concept of design of a structure with superior properties getting attention of the industry. Possible applications of CNTs have increased day by day.

CNTs can be modeled by using continuum mechanics. Atomic interactions like small scale effect, surface stresses and long distance interaction can not be ignored in the nano-dimensional mechanics. Eringen [1,2] dealt with this problem and proposed the Nonlocal Elasticity Theory which includes the size effect and has been used in the most of the recent researches about modeling of CNTs.

Most of the papers about statics and dynamics of CNTs assumed that a CNT is an elastic structure. However, damping characteristics of CNT structures should be accounted in the continuum model for more realistic approach.

Lei et al. investigated the dynamic behavior of nonlocal viscoelastic Euler-Bernoulli [3] and Timoshenko nanobeams [4]. Dynamic stability and buckling of viscoelastic nanobeams studied by Chen et al. [5] and Pavlovic et al. [6]. Buckling of cantilever nanotubes [7,8], boron-nitride nanotubes [9] and silicon-carbide nanotubes [10] investigated by researchers. Karlicic et al. [11] carried out the free transverse vibration analysis of the multiple CNTs embedded in a viscoelastic polymer matrix which was affected by an axial magnetic field. Arani et al. [12] investigated the free and forced vibrations of double viscoelastic piezoelectric nanobeams with 
the help of nonlocal viscoelasticity. Mohammadi [13] studied the vibration of rotating viscoelastic nanobeam with the thermal and humidity effect. Zhang et al. [14] investigated the transverse vibration of an axially loaded viscoelastic nanobeam embedded in elastic medium. Ebrahimi and Barati used the nonlocal strain gradient theory for the viscoelastic functionally graded (FG) nanobeams which resting on viscoelastic medium in the analysis of problems like: free vibration [15], hygro-thermal loading [16,17], surface and thermal effects [18] and size effect of nano-grains and nano-voids [19]. Attia and Mahmoud [20] modeled the viscoelastic nanobeam by using nonlocal couple-stress elasticity. Attia and Abdel Rahman [21] studied the free vibration of a FG viscoelastic nanobeams including the rotation and surface energy effects. Also, fractional nonlocal elasticity models have been proposed for dynamic analysis of viscoelastic nanobeams in recent studies [22-27] .

Present work assumes the CNT structure as a Kelvin-Voigt type viscoelastic material. Axial load effect through the buckling including nonlocal effect and viscoelasticity will be investigated. Variation of the non-dimensional frequency and damping of the nanobeam will be depicted in figures. Critical buckling load characteristics will be obtained.

\section{Analysis}

Viscoelastic nanobeams with simply supported and clamped-free boundary conditions are considered (Fig. (1)). The governing equation of motion for an axially loaded viscoelastic nanobeam can be interpreted as [28]:

$$
E\left(1+\alpha \frac{\partial}{\partial t}\right) I \frac{\partial^{4} w(x, t)}{\partial x^{4}}=-m \frac{\partial^{2} w(x, t)}{\partial t^{2}}-P \frac{\partial^{2} w(x, t)}{\partial x^{2}}
$$

where $E$ is the Young's modulus, $m$ is the mass per unit length, $I$ is the moment of inertia, $w$ is the transverse displacement of the CNT, $\alpha$ is the viscous parameter of the viscoelastic material and $P$ is the axial load.

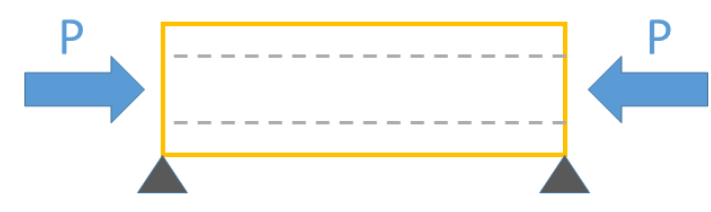

(a)

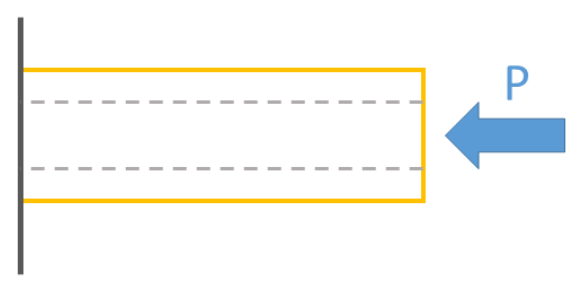

(b)

Fig. 1. Continuum Model of the Present Problem: a) Simply Supported Nanobeam b)Clamped-Free Nanobeam

\subsection{Nonlocal Elasticity Approach}

Nonlocal constitutive stress-strain relation can be expressed in differential form as [29]:

$$
\left(1-\mu \nabla^{2}\right) \tau_{k l}=\lambda \varepsilon_{r r} \delta_{k l}+2 G \varepsilon_{k l}
$$

where $\tau_{k l}$ is the nonlocal stress tensor, $\delta_{k l}$ is the strain tensor, $\lambda$ and $G$ are the material constants and $\mu=\left(e_{0} a\right)^{2}$ is called nonlocal parameter. Eringen obtained very close results to discrete theory results with a nonlocal continuum approach. With Eringen's assumption, the nonlocal model comprises both discrete and continuum approaches. 
For isotropic viscoelastic carbon nanotube, nonlocal one dimensional relation in axial direction can be written as:

$$
\left(1-\mu \frac{\partial^{2}}{\partial x^{2}}\right) \sigma=E\left(1+\alpha \frac{\partial}{\partial t}\right) \varepsilon
$$

where $\varepsilon$ and $\sigma$ are the normal strain and the normal stress, respectively.

\subsection{Nonlocal Equation of Motion and Boundary Conditions}

If Eq. (3) is inserted into Eq. (1), one obtains [30,31]:

$$
E\left(1+\alpha \frac{\partial}{\partial t}\right) I \frac{\partial^{4} w(x, t)}{\partial x^{4}}=-\left(1-\mu \frac{\partial^{2}}{\partial x^{2}}\right)\left(m \frac{\partial^{2} w(x, t)}{\partial t^{2}}+P \frac{\partial^{2} w(x, t)}{\partial x^{2}}\right)
$$

If Eq. (4) is reorganized according to D'Alambert Principle:

$$
E I \alpha \frac{\partial^{5} w(x, t)}{\partial x^{4} \partial t}+E I \frac{\partial^{4} w(x, t)}{\partial x^{4}}+m \frac{\partial^{2} w(x, t)}{\partial t^{2}}+P \frac{\partial^{2} w(x, t)}{\partial x^{2}}-\mu m \frac{\partial^{4} w(x, t)}{\partial x^{2} \partial t^{2}}-\mu P \frac{\partial^{4} w(x, t)}{\partial x^{4}}=0
$$

Eq. (5) is the equation of motion of a viscoelastic nanobeam. If the nonlocal parameter and viscoelasticity parameter are assumed to be zero $(\mu=0, \alpha=0)$, the classical elasticity equation will be obtained. The boundary condition on both edges of nanobeam considered as simply supported (S-S) and clamped-free (C-F) which are defined below [32]:

$$
\begin{gathered}
x=0\left\{\begin{array}{l}
w(0, t)=0 \\
-E\left(1+\alpha \frac{\partial}{\partial t}\right) I \frac{\partial^{2} w(0, t)}{\partial x^{2}}+\mu P \frac{\partial^{2} w(0, t)}{\partial x^{2}}+\mu m \frac{\partial^{2} w(0, t)}{\partial t^{2}}=0
\end{array}\right\} S-S \\
x=L\left\{\begin{array}{l}
w(L, t)=0 \\
-E\left(1+\alpha \frac{\partial}{\partial t}\right) I \frac{\partial^{2} w(L, t)}{\partial x^{2}}+\mu P \frac{\partial^{2} w(L, t)}{\partial x^{2}}+\mu m \frac{\partial^{2} w(L, t)}{\partial t^{2}}=0
\end{array}\right) \\
x=0\left\{\begin{array}{l}
w(0, t)=0 \\
\frac{\partial w(0, t)}{\partial x}=0
\end{array}\right\} C-F \\
x=L\left\{\begin{array}{l}
-E\left(1+\alpha \frac{\partial}{\partial t}\right) I \frac{\partial^{2} w(L, t)}{\partial x^{2}}+\mu P \frac{\partial^{2} w(L, t)}{\partial x^{2}}+\mu m \frac{\partial^{2} w(L, t)}{\partial t^{2}}=0 \\
-E\left(1+\alpha \frac{\partial}{\partial t}\right) I \frac{\partial^{3} w(L, t)}{\partial x^{3}}-P \frac{\partial w(L, t)}{\partial x}+\mu P \frac{\partial^{3} w(L, t)}{\partial x^{3}}+\mu m \frac{\partial^{3} w(L, t)}{\partial x \partial t^{2}}=0
\end{array}\right\}
\end{gathered}
$$

The transverse displacement $w$ can be expressed as:

$$
w(x, t)=A(x) e^{\lambda t}
$$

where $A(x)$ and $\lambda$ is the amplitude function and characteristic value for viscoelastic nanobeam vibration, respectively. Inserting Eq. (8) into Eq. (5) gives following dimensionless equations of motion with the assumption of dimensionless nanotube length $\left(\bar{x}=\frac{x}{L}\right)$ :

$$
\frac{\partial^{4} A(\bar{x})}{\partial \bar{x}^{4}}\left(1+\alpha \lambda-\frac{\mu}{L^{2}} \bar{P}\right)+\frac{\partial^{2} A(\bar{x})}{\partial \bar{x}^{2}}\left(\bar{P}-\frac{\mu}{L^{2}} \Omega \lambda^{2}\right)+A(\bar{x})\left(\Omega \lambda^{2}\right)=0
$$

where $\bar{P}$ is the dimensionless axial load and $\Omega$ is the characteristic parameter coefficient which are defined as below: 


$$
\bar{P}=\frac{P L^{2}}{E I} \quad, \quad \Omega=\frac{m L^{4}}{E I}
$$

Eq. (9) is a forth order differential equation and general solution can be written as:

$$
A(\bar{x})=C_{1} e^{r_{1} \bar{x}}+C_{2} e^{r_{2} \bar{x}}+C_{3} e^{r_{3} \bar{x}}+C_{4} e^{r_{4} \bar{x}}
$$

where $C_{i}$ and $r_{i}(i=1,2,3,4)$ are the integration constants and the roots of the characteristic equation in Eq. (9), respectively. In Eqs. (6) and (7), boundary conditions should be written in matrix form using amplitude function in Eq. (8) for unknown coefficients as below:

$$
\left[\begin{array}{llll}
P_{11} & P_{12} & P_{13} & P_{14} \\
P_{21} & P_{22} & P_{23} & P_{24} \\
P_{31} & P_{32} & P_{33} & P_{34} \\
P_{41} & P_{42} & P_{43} & P_{44}
\end{array}\right]\left[\begin{array}{l}
C_{1} \\
C_{2} \\
C_{3} \\
C_{4}
\end{array}\right]=0
$$

Eq. (12) is an eigen-value problem and the determinant of the coefficient matrix must be equal to zero for a nontrivial solution. Characteristic parameter $(\lambda)$ for the viscoelastic nanobeam vibration can be obtained from determinant equation. $\lambda$ is a complex number and its imaginary part defines the non-dimensional frequency (NDF) and real part defines the non-dimensional damping (NDD) of viscoelastic nanobeam.

Buckling is a structural stability loss and can be seen on axially loaded beams. It is a limit value problem that free vibration frequency of the structure drops to zero (See Eq. (13)).

$$
\lim _{N D F \rightarrow 0} \bar{P}=P_{C R}
$$

\section{Numerical Results and Discussion}

In this section, free transverse vibration analysis of the viscoelastic nanobeams has been carried out for various nonlocal parameter, viscous parameter and axial load.

Validation of the present nonlocal elastic CNT nanobeam model has been carried out in previous study [33]. Lattice dynamics results have been used in order to compare the nonlocal elastic stress gradient model. The nonlocal theory gives close results with the lattice dynamics results at the end of first Brillouin Zone.

In Fig. (2), the nonlocality and viscous effect on complex characteristic parameter of viscoelastic nanobeam in simply supported boundary condition can be seen. Softening effect of nonlocality has been addressed in previous works [34,35]. Nonlocal parameter reduces the NDF because of the softening. Viscous characteristics of the viscoelastic material also reduces with nonlocal parameter because nonlocality increases the elastic behavior of the material. Viscous parameter $(\alpha)$ decreases the NDF and increases the NDD. That is an expected result from the classical continuum mechanics approach. Clamped-free boundary condition results are shown in Fig. (3). Except one case, clamped-free boundary conditions gives same results with simply supported boundary case. In contrary to S-S case, clamped-free boundary condition increase the NDD and nanobeam buckles easily for higher nonlocal parameters. With applying compressive axial load, nanobeam can buckle easily in C-F boundary case and this situation can be seen clearly in Fig. (7). 
In Figs. (4) and (5), axial compressive load effect on complex characteristic parameter of viscoelastic nanobeam can be seen. Axial load reduces the NDF and has no effect on NDD in simply supported boundary case. On the other hand, axial load change the vibration characteristics in clamped-free boundary case. Without the axial load, NDF and NDD increases with the help of nonlocality. With the axial load, NDF decreases and NDD increases reversely.

In the vibration of clamped-free nanobeams, nonlocal effect shows a strengthening effect on the material and fundamental frequency of nanobeam increases in contrary to other boundary conditions. This phenomena discussed by scientists in several studies [36-38] . Li et al. [39,40] pointed out that, both enhancing and weakening nonlocal effects are possible and correct. Nonlocal integral models have been used in recent studies to overcome this paradox [41-43].

Buckling of viscoelastic nanobeams can be seen in Figs. (6) and (7). NDF drops to zero with increasing effect of axial load. NDD doesn't change in S-S case but increases in C-F case with axial load. Viscoelasticity increases the NDF with nonlocal elasticity approach for a nanobeam without an axial load applied. This result is due to the nonlocal viscoelastic effects and contradicts with nonlocal elastic beam model.

Variation of critical buckling load is shown in Fig. (8) for the both boundary cases. Critical buckling load changes only with nonlocal parameter. Nonlocal parameters reduces CBL whereas viscous parameter couldn't change it.
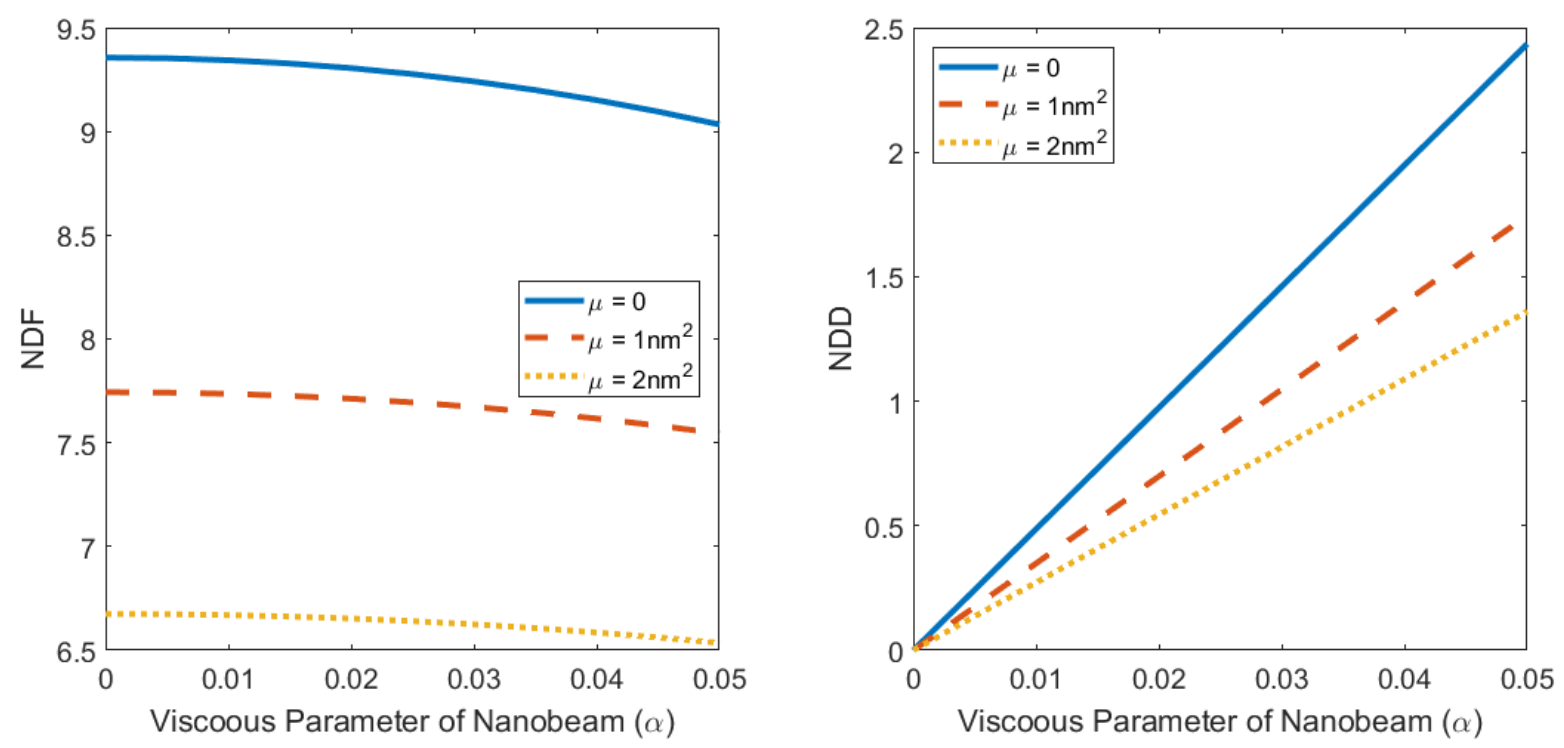

Fig. 2. Nonlocal and Viscous Parameter Effects on NDF and NDD in Simply Supported Boundary Condition $(\overline{\mathrm{P}}=1)$ 

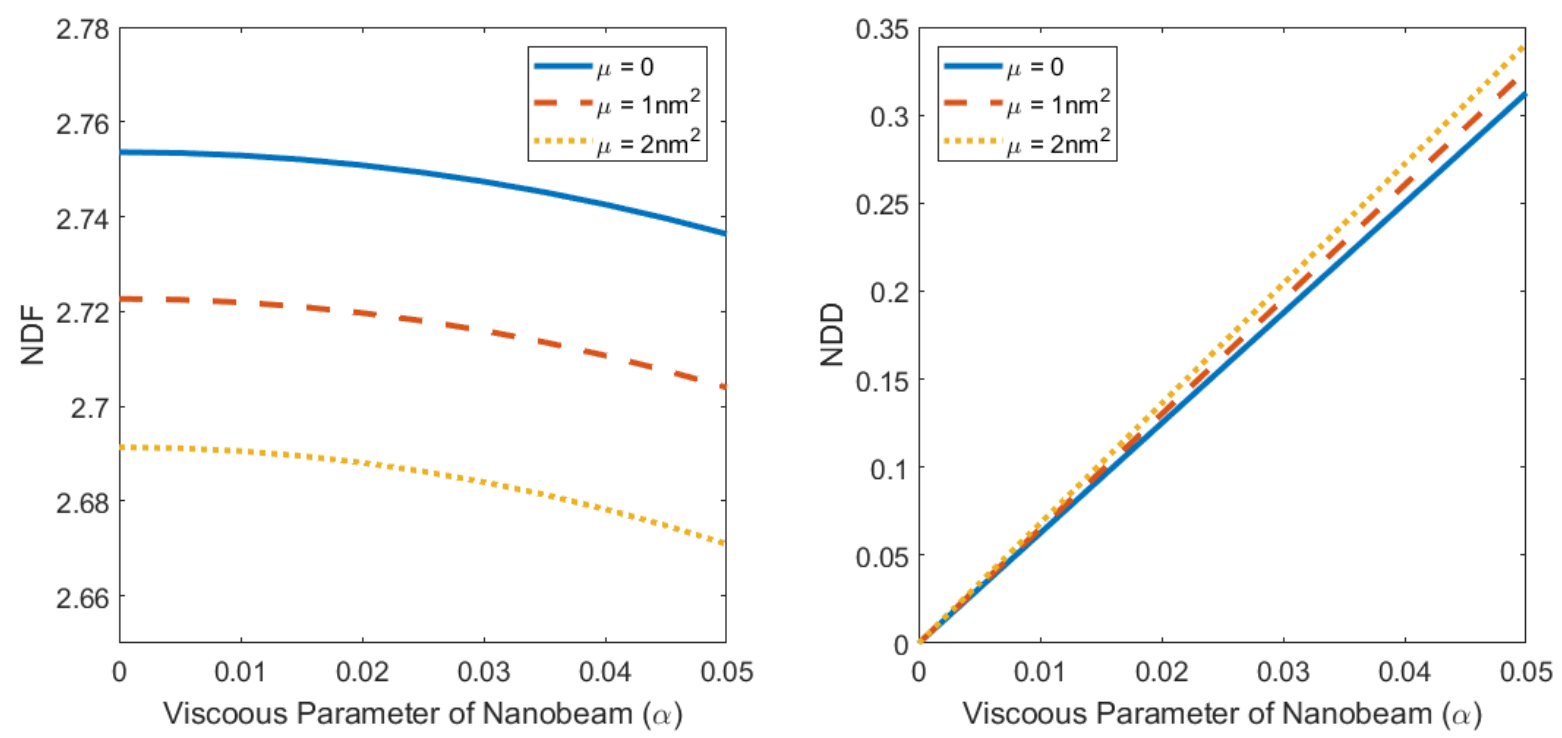

Fig. 3. Nonlocal and Viscous Parameter Effects on NDF and NDD in Clamped-Free Boundary Condition $(\overline{\mathrm{P}}=1)$
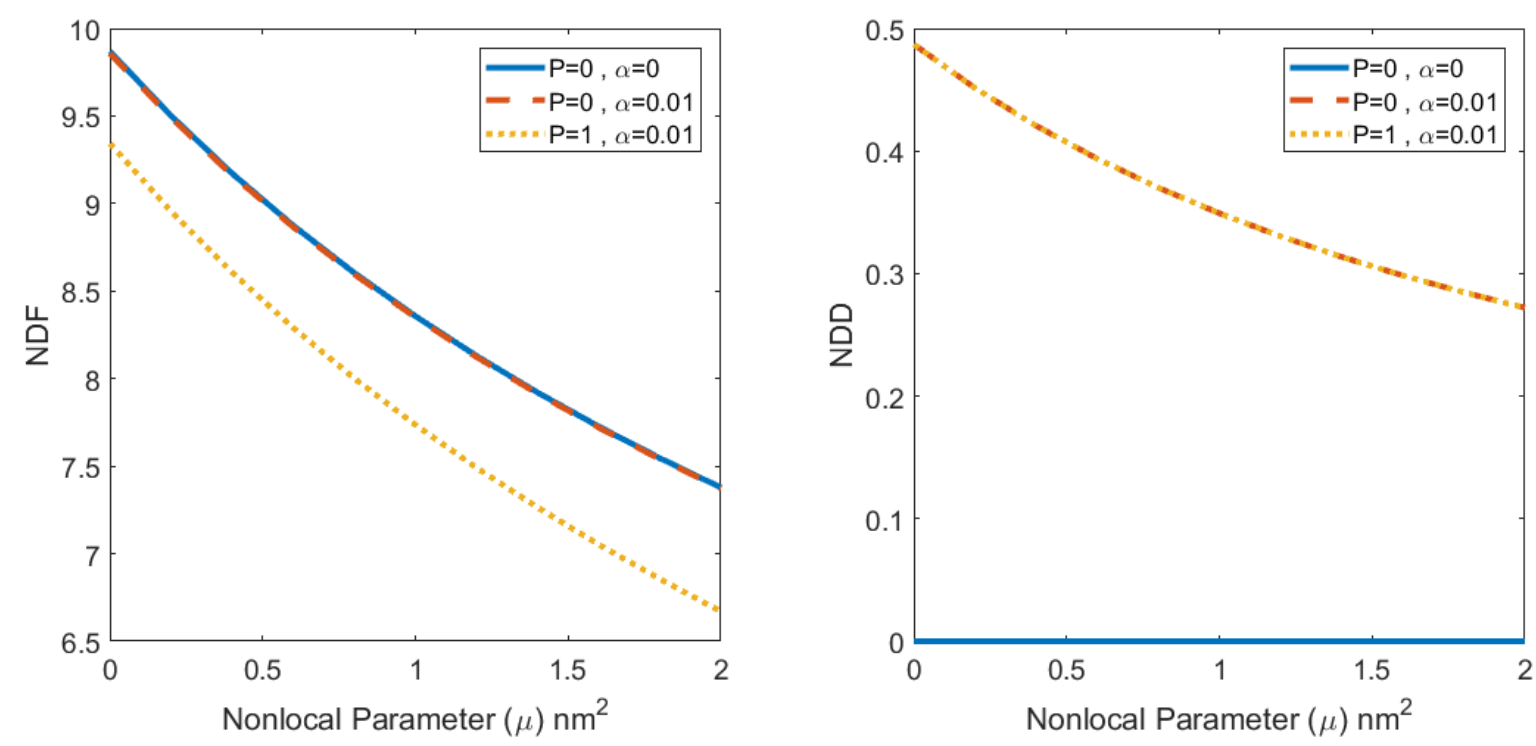

Fig. 4. Nonlocal, Viscous and Axial Load Effects on NDF and NDD in Simply Supported Boundary Condition 

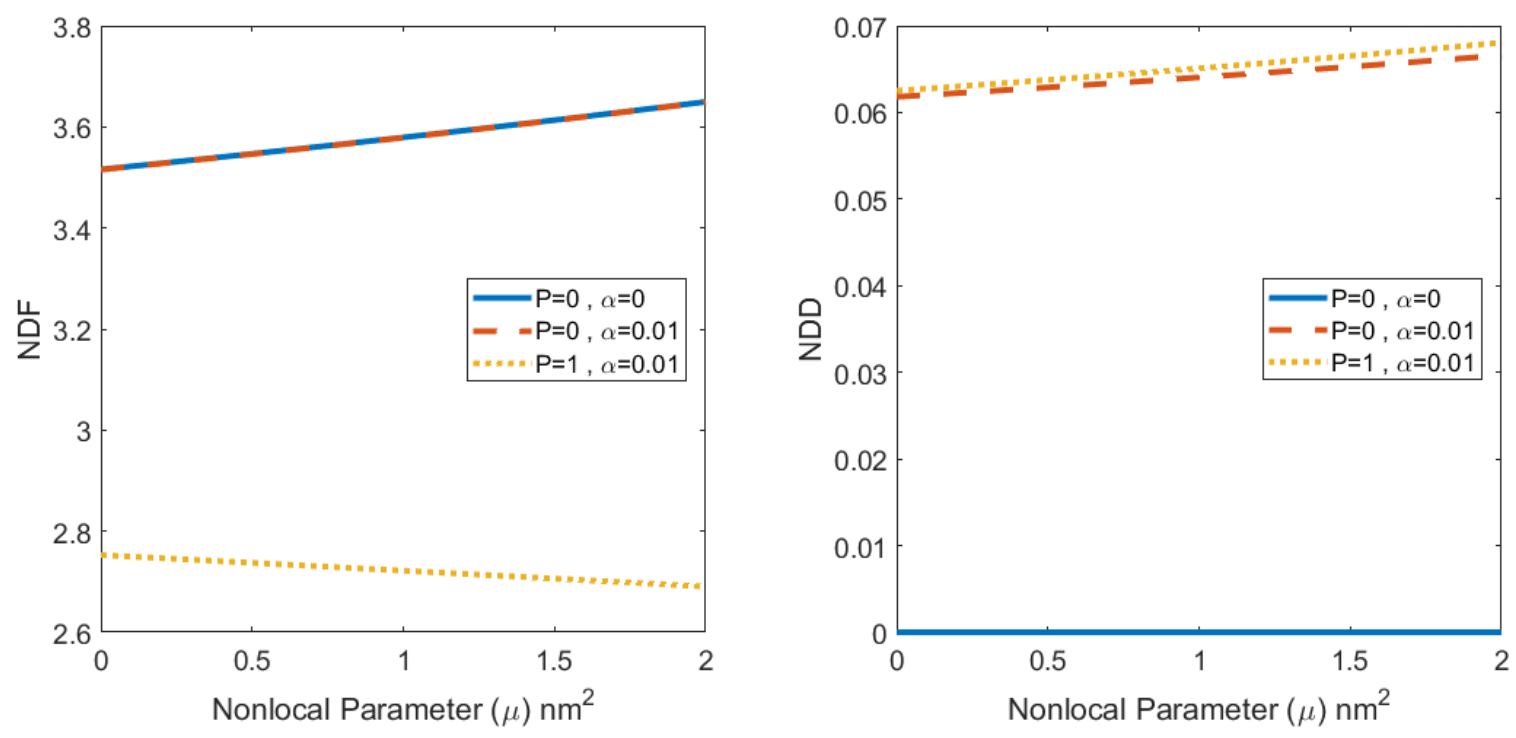

Fig. 5. Nonlocal, Viscous and Axial Load Effects on NDF and NDD in Clamped-Free Boundary Condition
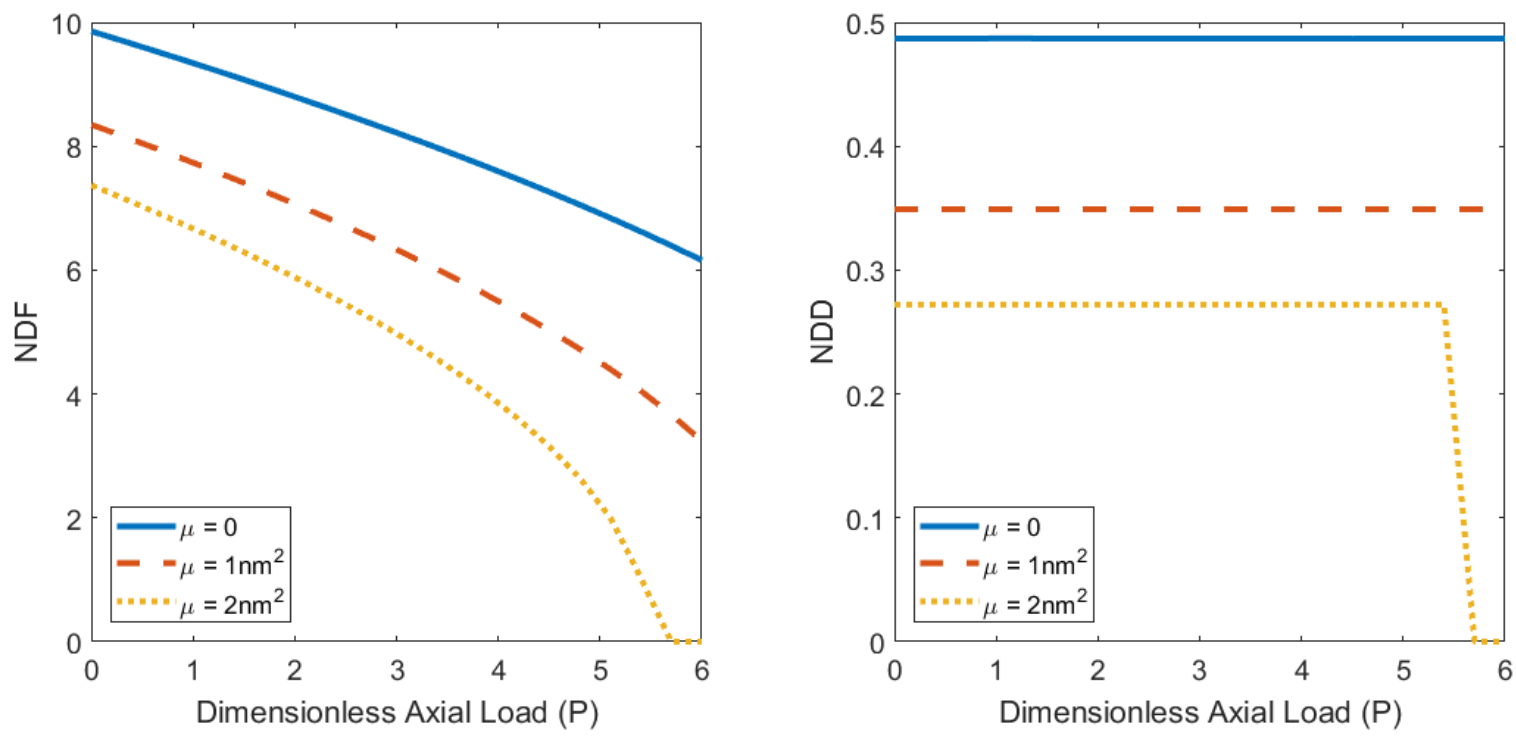

Fig. 6. Nonlocal Effect on Buckling of Viscoelastic Nanobeam in Simply Supported Boundary Condition $(\alpha=0.01)$ 

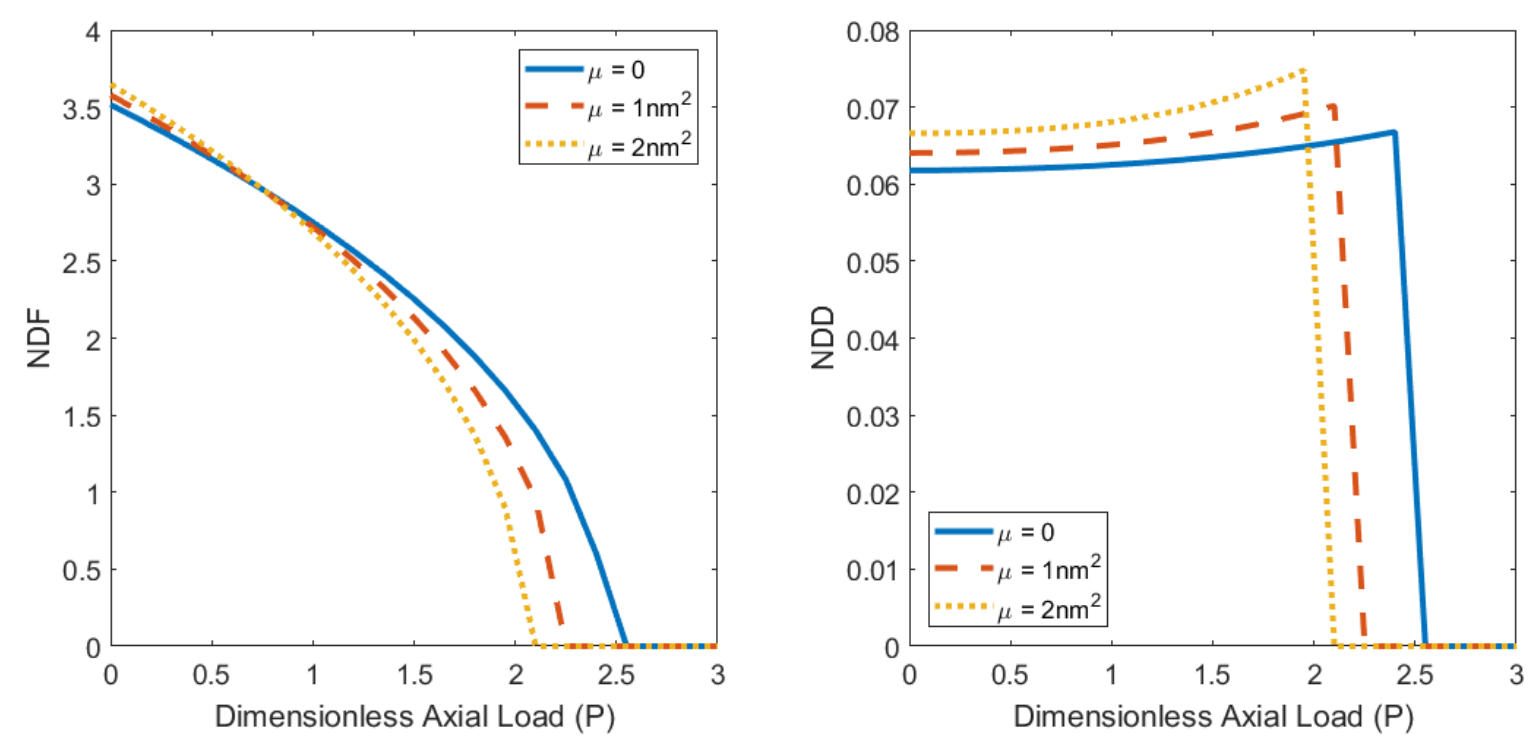

Fig. 7. Nonlocal Effect on Buckling of Viscoelastic Nanobeam in Clamped-Free Boundary Condition $(\alpha=0.01)$
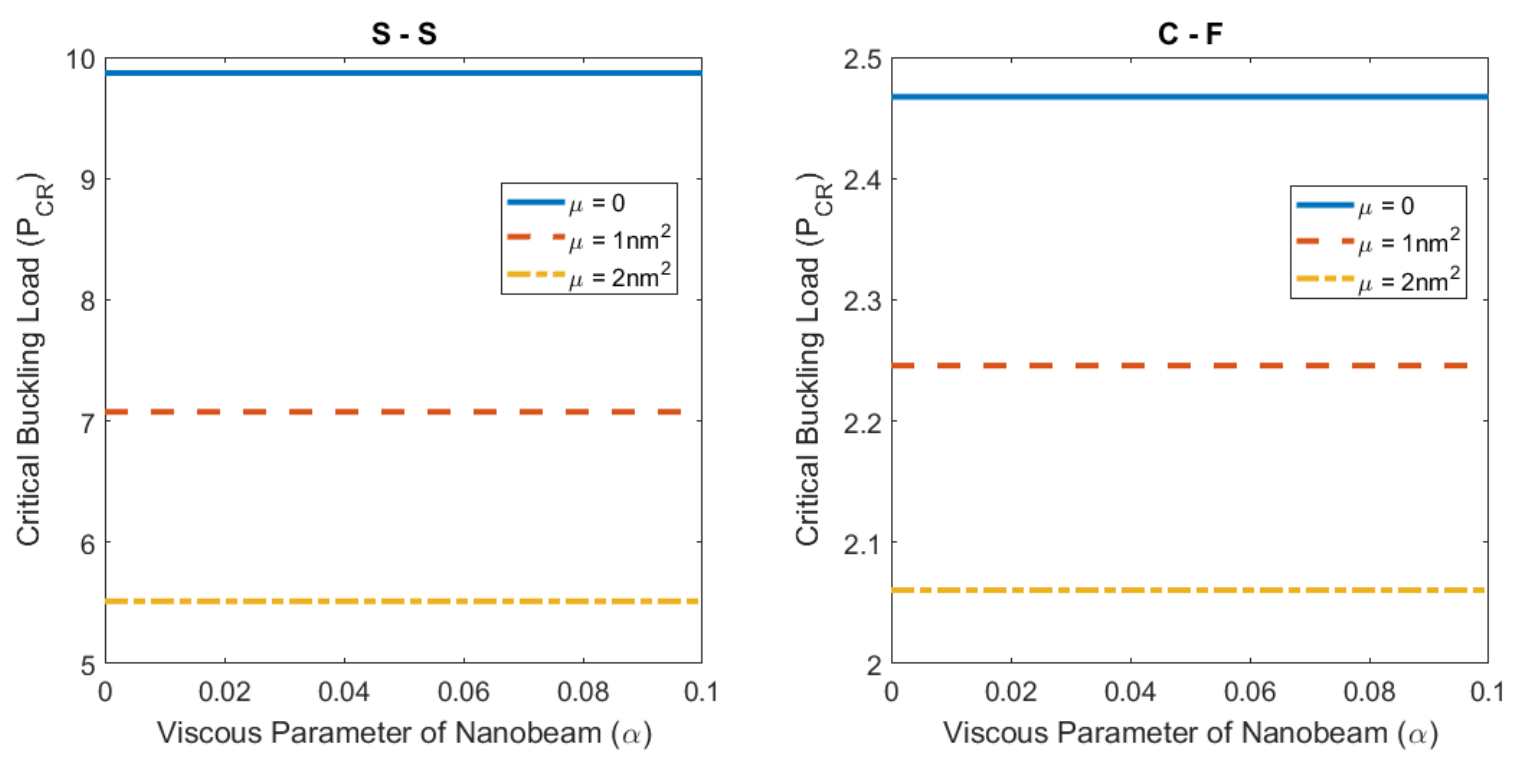

Fig. 8. Variation of Critical Buckling Load with Nonlocal and Viscous Parameters (NDF=NDD=0)

\section{Conclusion}

Present study deals with the vibration problem of an axially loaded viscoelastic nanobeam with simply supported and clamped-free boundary conditions. The nanobeam has been assumed as Kelvin-Voigt type viscoelastic material. Governing equations and boundary conditions have obtained with Eringen's Nonlocal Elasticity Theory. The viscous effect of viscoelastic medium decreases the complex characteristic parameter of nanobeam in simply supported boundary case. But in clamped-free boundary case, viscous effect increases the complex characteristic parameter because of the nonlocal boundary condition. Axial load and nonlocal effect shows 
softening effect on nanobeam lattice structure. Results could be useful in designing a nanomass sensor applications.

\section{References}

[1] Eringen A.C., On differential equations of nonlocal elasticity and solutions of screw dislocation and surface waves, Journal of Applied Physics, 54, 4703-10, 1983. doi:10.1063/1.332803

[2] Eringen A.C., Nonlocal polar elastic continua, International Journal of Engineering Science, 10, 1-16, 1972. doi:10.1016/0020-7225(72)90070-5

[3] Lei Y., Murmu T., Adhikari S., Friswell M.I., Dynamic characteristics of damped viscoelastic nonlocal Euler-Bernoulli beams, European Journal of Mechanics, A/Solids, 42, 125-36, 2013. doi:10.1016/j.euromechsol.2013.04.006

[4] Lei Y., Adhikari S., Friswell M.I., Vibration of nonlocal Kelvin-Voigt viscoelastic damped Timoshenko beams, International Journal of Engineering Science, 66-67, 1-13, 2013. doi:10.1016/j.ijengsci.2013.02.004

[5] Chen C., Li S., Dai L., Qian C., Buckling and stability analysis of a piezoelectric viscoelastic nanobeam subjected to van der Waals forces, Communications in Nonlinear Science and Numerical Simulation, 19, 1626-37, 2014. doi:10.1016/j.cnsns.2013.09.017

[6] Pavlović I., Pavlović R., Ćirić I., Karličić D., Dynamic stability of nonlocal Voigt-Kelvin viscoelastic Rayleigh beams, Applied Mathematical Modelling, 39, 6941-50, 2015. doi:10.1016/j.apm.2015.02.044

[7] Civalek Ö., Demir C., Buckling and bending analyses of cantilever carbon nanotubes using the Euler-Bernoulli beam theory based on non-local continuum model, Asian Journal of Civil Engineering, 12, 651-62, 2011

[8] Akgöz B., Civalek Ö., Buckling Analysis of Cantilever Carbon Nanotubes Using the Strain Gradient Elasticity and Modified Couple Stress Theories, Journal of Computational and Theoretical Nanoscience, 8, 1821-7, 2011. doi:10.1166/jctn.2011.1888

[9] Mercan K., Civalek Ö., DSC method for buckling analysis of boron nitride nanotube (BNNT) surrounded by an elastic matrix, Composite Structures, 143, 300-9, 2016. doi:10.1016/j.compstruct.2016.02.040

[10] Mercan K., Civalek Ö., Buckling analysis of Silicon carbide nanotubes (SiCNTs) with surface effect and nonlocal elasticity using the method of HDQ, Composites Part B: Engineering, 114, 34-45, 2017. doi:10.1016/j.compositesb.2017.01.067

[11] Karličić D., Murmu T., Cajić M., Kozić P., Adhikari S., Dynamics of multiple viscoelastic carbon nanotube based nanocomposites with axial magnetic field, Journal of Applied Physics, 115, 234303, 2014. doi:10.1063/1.4883194

[12] Ghorbanpour-Arani A.H., Rastgoo A., Sharafi M.M., Kolahchi R., Ghorbanpour Arani A., Nonlocal viscoelasticity based vibration of double viscoelastic piezoelectric 
nanobeam systems, Meccanica, 51, 25-40, 2016. doi:10.1007/s11012-014-9991-0

[13] Mohammadi M., Safarabadi M., Rastgoo A., Farajpour A., Hygro-mechanical vibration analysis of a rotating viscoelastic nanobeam embedded in a visco-Pasternak elastic medium and in a nonlinear thermal environment, Acta Mechanica, 227, 2207-32, 2016. doi:10.1007/s00707-016-1623-4

[14] Zhang Y., Pang M., Fan L., Analyses of transverse vibrations of axially pretensioned viscoelastic nanobeams with small size and surface effects, Physics Letters, Section A: General, Atomic and Solid State Physics, 380, 2294-9, 2016. doi:10.1016/j.physleta.2016.05.016

[15] Ebrahimi F., Barati M.R., Vibration analysis of viscoelastic inhomogeneous nanobeams incorporating surface and thermal effects, Applied Physics A: Materials Science and Processing, 123, 1-10, 2017. doi:10.1007/s00339-016-0511-z

[16] Ebrahimi F., Barati M.R., Hygrothermal effects on vibration characteristics of viscoelastic FG nanobeams based on nonlocal strain gradient theory, Composite Structures, 159, 433-44, 2017. doi:10.1016/j.compstruct.2016.09.092

[17] Ebrahimi F., Barati M.R., Effect of three-parameter viscoelastic medium on vibration behavior of temperature-dependent non-homogeneous viscoelastic nanobeams in a hygro-thermal environment, Mechanics of Advanced Materials and Structures, 25, 36174, 2018. doi:10.1080/15376494.2016.1255831

[18] Ebrahimi F., Barati M.R., Vibration analysis of viscoelastic inhomogeneous nanobeams resting on a viscoelastic foundation based on nonlocal strain gradient theory incorporating surface and thermal effects, Acta Mechanica, 228, 1197-210, 2017. doi:10.1007/s00707$016-1755-6$

[19] Ebrahimi F., Barati M.R., Damping Vibration Behavior of Viscoelastic Porous Nanocrystalline Nanobeams Incorporating Nonlocal-Couple Stress and Surface Energy Effects, Iranian Journal of Science and Technology, Transactions of Mechanical Engineering, 2017. doi:10.1007/s40997-017-0127-8

[20] Attia M.A., Mahmoud F.F., Analysis of viscoelastic Bernoulli-Euler nanobeams incorporating nonlocal and microstructure effects, International Journal of Mechanics and Materials in Design, 13, 385-406, 2017. doi:10.1007/s10999-016-9343-4

[21] Attia M.A., Abdel Rahman A.A., On vibrations of functionally graded viscoelastic nanobeams with surface effects, International Journal of Engineering Science, 127, 132, 2018. doi:10.1016/j.ijengsci.2018.02.005

[22] Oskouie M.F., Ansari R., Linear and nonlinear vibrations of fractional viscoelastic Timoshenko nanobeams considering surface energy effects, Applied Mathematical Modelling, 43, 337-50, 2017. doi:10.1016/j.apm.2016.11.036

[23] Oskouie M.F., Ansari R., Sadeghi F., Nonlinear vibration analysis of fractional viscoelastic Euler-Bernoulli nanobeams based on the surface stress theory, Acta Mechanica Solida Sinica, 30, 416-24, 2017. doi:10.1016/j.camss.2017.07.003

[24] Ansari R., Faraji Oskouie M., Rouhi H., Studying linear and nonlinear vibrations of 
fractional viscoelastic Timoshenko micro-/nano-beams using the strain gradient theory, Nonlinear Dynamics, 87, 695-711, 2017. doi:10.1007/s11071-016-3069-6

[25] Ansari R., Faraji Oskouie M., Gholami R., Size-dependent geometrically nonlinear free vibration analysis of fractional viscoelastic nanobeams based on the nonlocal elasticity theory, Physica E: Low-Dimensional Systems and Nanostructures, 75, 266-71, 2016. doi:10.1016/j.physe.2015.09.022

[26] Ansari R., Faraji Oskouie M., Sadeghi F., Bazdid-Vahdati M., Free vibration of fractional viscoelastic Timoshenko nanobeams using the nonlocal elasticity theory, Physica E: Low-Dimensional Systems and Nanostructures, 74, 318-27, 2015. doi:10.1016/j.physe.2015.07.013

[27] Cajic M., Karlicic D., Lazarevic M., Nonlocal vibration of a fractional order viscoelastic nanobeam with attached nanoparticle, Theoretical and Applied Mechanics, 42, 167-90, 2015. doi:10.2298/TAM1503167C

[28] Marynowski K., Non-Linear Dynamic Analysis of an Axialy Moving Viscoelastic Beam, Journal of Theoretical and Applied Mechanics, 465-82, 2002

[29] Eringen A.C., Nonlocal Continuum Field Theories. Springer New York, 2007

[30] Civalek Ö., Demir Ç., Akgöz B., Static analysis of single walled carbon nanotubes (SWCNT) based on Eringen's nonlocal elasticity theory, International Journal of Engineering and Applied Sciences, 1, 47-56, 2009

[31] Akgöz B., Civalek Ö., Investigation of Size Effects on Static Response of Single-Walled Carbon Nanotubes Based on Strain Gradient Elasticity, International Journal of Computational Methods, 09, 1240032, 2012. doi:10.1142/S0219876212400324

[32] Reddy J.N., Pang S.D., Nonlocal continuum theories of beams for the analysis of carbon nanotubes, Journal of Applied Physics, 103, 2008. doi:10.1063/1.2833431

[33] Aydogdu M., A general nonlocal beam theory: Its application to nanobeam bending, buckling and vibration, Physica E: Low-Dimensional Systems and Nanostructures, 41, 1651-5, 2009. doi:10.1016/j.physe.2009.05.014

[34] Arda M., Aydogdu M., Buckling of Eccentrically Loaded Carbon Nanotubes, Solid State Phenomena, 267, 151-6, 2017. doi:10.4028/www.scientific.net/SSP.267.151

[35] Arda M., Aydogdu M., Nonlocal Gradient Approach on Torsional Vibration of CNTs, NOISE Theory and Practice, 3, 2-10, 2017

[36] Lu P., Lee H.P., Lu C., Zhang P.Q., Dynamic properties of flexural beams using a nonlocal elasticity model, Journal of Applied Physics, 99, 073510, 2006. doi:10.1063/1.2189213

[37] Eltaher M.A., Alshorbagy A.E., Mahmoud F.F., Vibration analysis of Euler-Bernoulli nanobeams by using finite element method, Applied Mathematical Modelling, 37, 478797, 2013. doi:10.1016/j.apm.2012.10.016

[38] Romano G., Barretta R., Diaco M., Marotti de Sciarra F., Constitutive boundary 
conditions and paradoxes in nonlocal elastic nanobeams, International Journal of Mechanical Sciences, 121, 151-6, 2017. doi:10.1016/j.ijmecsci.2016.10.036

[39] Li C., A nonlocal analytical approach for torsion of cylindrical nanostructures and the existence of higher-order stress and geometric boundaries, Composite Structures, 118, 607-21, 2014. doi:10.1016/j.compstruct.2014.08.008

[40] Li C., Torsional vibration of carbon nanotubes: Comparison of two nonlocal models and a semi-continuum model, International Journal of Mechanical Sciences, 82, 25-31, 2014. doi:10.1016/j.ijmecsci.2014.02.023

[41] Challamel N., Reddy J.N., Wang C.M., Eringen's Stress Gradient Model for Bending of Nonlocal Beams, Journal of Engineering Mechanics, 142, 04016095, 2016. doi:10.1061/(ASCE)EM.1943-7889.0001161

[42] Eptaimeros K.G., Koutsoumaris C.C., Tsamasphyros G.J., Nonlocal integral approach to the dynamical response of nanobeams, International Journal of Mechanical Sciences, 115-116, 68-80, 2016. doi:10.1016/j.ijmecsci.2016.06.013

[43] Shaat M., Faroughi S., Abasiniyan L., Paradoxes of differential nonlocal cantilever beams: Reasons and a novel solution, 1-17, 2017 